\title{
WATER SYSTEM COMPLEXITY AND THE MISUSE OF MODELING AND OPTIMIZATION
}

\author{
Yacov Y. Haimes \\ Center for Risk Management of Engineering Systems \\ Univer sity of Virg inia
}

\section{PERSONAL BACKGROUND}

Pursuing my graduate work at University of California, Los Angeles (UCLA) in 1965, I received a research assistantship from W arren A. Hall, then Director of the Water Resources Center, and later my close friend and collaborator. Although both my graduate degrees are in Systems Engineering, my interest in the modeling, planning, and management of water resources systems has always inspired $m$ y profess ional gro wth and contributions.

\section{INTRODUCTION}

The complexity of water and related land system s, which is due primarily to their large number of constituencies and interdependent subsystems, is familiar to all those practicing in the field. In our quest to model this complexity, however, we have over the years developed and adopted relatively manageable models that often oversimplify some fundamental attributes of these systems. Most water distribution networks consist of a vast number of interconnected components - e.g., the distribution network, pumps, pipes, and treatment plants. In addition, a hierarchy of institutional and organizational structures - e.g., federal, state, county, and city - is involved in the decisionmaking process. The degree of physical and institutional coup ling that exists among the subsystems (e.g., the budget constraint imposed on the overall system) fu rther com plicates their modeling as well as manag ement. In the maintenance of water distribution systems, different replacement/repair strategies for varying subsystems often have unexpected impacts on the overall system; the demands for the resources and the ir approp riate allocations likewise have a diverse impact on the system 's reliability.

The following statement seems as relevant today as it was two decades ago (Haimes 1977):

In studying large-scale systems with techn ological, societal, and environmental aspects, the efforts in the modeling as well as in the optimization (solution of the system model) are magnified and often overwhelm the analysis. This is due to the high dimensionality (very large number of variables) and complexity (non-linearity in the coupling and interactions among the variables) of the resulting models.

When facing such a complex modeling task, it is natural to tend to aggregation and to reductionist modeling tools. Aggregation as sumessufficiently common characteristics among the components to merit linking them in one class or category. Indeed, the essence of modeling consists of selecting the appropriate level of aggregation and reduction, modeling tools, time scale, physical scale, system boundary, model topology (e.g., level of nonlinearity), model param eters, represe ntative objectives and constraints, the appropriate visions of the systems that should be modeled, and the appropriate metrics upon which such models are built.

This paper will focus on five points. These are the flaws of four metrics when used in modeling water resources systems without discrimination, plus the unqualified use of model optimization as a surrogate to system optimization:

- Cost-be nefit analysis as a surrogate for genuine tradeoffs among multiple noncommensurate cost, bene fit, and risk objectives.

- Expected value of risk.

- Present value of money.

- Reliability analysis as a surrog ate to risk analysis.

- The fallacy of optimization.

The flaws in the metrics stem from their precommensurating inherent multiple objectives of different dimensions by lumping them into a single objective, and thus curtailing mandated explicit trade-off analyses. These overly simplified metricshave becomeso entrenched as measu res of efficien cy and e ffectiveness in the fabric of our analyses, that we commonly use them without much discrimination and do not repeatedly question their appropriatene ss or representativeness. This paper aims to demonstrate that the first three of the four metrics essentially convert inherent multiobjective problems into a single objective one, and the fourth m etric avoids explicit trade-offs altogether. The last point reflects on the misuse of optimization in decisionmaking. 


\section{COST-BENEFIT ANALYSIS AS A SURROGATE FOR GENUINE TRADE-OFFS AMONG MULTIPLE NONCOMMENSURATE OBJECTIVE S OF COSTS, BENEFITS, AND RISKS}

Consider the following three representative waterplanning objectives for the Maumee River Basin study. The fine-textured glacial tills and lake-deposited clays of the basin have poor natural drainage, and the soil's slow permeability, intensive row cropping, and urban sprawl are major contributors to soil erosion during heavy rains (Haim es 1977):

$$
\text { Minimize }\left\{\mathrm{f}_{1}(\mathbf{x}), \mathrm{f}_{2}(\mathbf{x}), \mathrm{f}_{3}(\mathbf{x})\right\}
$$

where $\mathbf{x}$ is a vector of policy options, $f_{1}(\mathbf{x})$ is the cost of plan implem entation [dollars], $f_{2}(\mathbf{x})$ is soil erosion in the basin [tons], and $\mathrm{f}_{3}(\mathbf{x})$ is risk of flooded land [acres]. Clearly, a multiobjective framework, where Pareto optimality and trade-offs dominate the analysis, is the most appropriate method for (1) as indeed w as the case in the Maumee study. A single-objective analysis, on the other hand, would replace the three objectives with one single metric by introduc ing weights to the system shown in (1):

$$
\begin{aligned}
& \text { Minimize }\left\{\mathrm{p}_{1} \mathrm{f}_{1}(\mathbf{x})+\mathrm{p}_{2} \mathrm{f}_{2}(\mathbf{x})+\mathrm{p}_{3} \mathrm{f}_{3}(\mathbf{x})\right\} \\
& \text { Whe re, } \mathrm{p}_{1}+\mathrm{p}_{2}+\mathrm{p}_{3}=1, \mathrm{p}_{\mathrm{I}} \quad 0, \quad \mathrm{i}=1,2,3
\end{aligned}
$$

Note that the Maumee planning board was much more concerned with the relative value of addition al increm ents of the three noncommensurable objectives, at a given value of each objective function, than it was with their absolute values. Furthermore, given a ny current set of objective levels attained, it is much more meaningful and effective for a planning board to assess the relative value of the trade-off of the marginal increases and decreases between any two objectives than it is to assess their absolute average values. Indeed, this view was endorsed by participants from 42 countries who attended the $14^{\text {th }}$ Conference of the International Society for Multiple Criteria Decision Making (MCDM), which was hosted at the University of Virginia in June 1998. They presented over 100 papers on the ever-growing importance of the MCD M field.

Single-objective analysis can be particularly flawed and misleading, if not totally erroneous, when risk (a measure of the probability and severity of adverse effects) is traded off with the cost of risk manage ment. This is because safety, the level of a cceptable risk, is not absolute; it must be traded off with the corresponding cost of risk reduction (mana gement) as well as with other objectives on relative as well as absolute values. Equation (2) fails to provide these imperativequintes sentialtrade-offs. Although many water experts have pioneered the use of multiple objectives in general and in water resources planning and management in particular, many studies rem ain hostage to the single-objective paradigm when multiobjective analyses are warranted.

\section{EXPECTE D VALUE OF RISK}

Risk is a measure of the probability and severity of adverse effects. One of the most dominant steps in the risk-assessment process is the quantification of risk, yet the validity of the expected value, the metric most comm only used to quantify risk, has received neither the broad professional scrutiny it deserves nor the hoped-for wider mathematicalchallenge that it mandates. Consider, for example, the concentration of the contaminant trichloroethylene (TCE) in a ground water system, measured in parts per billion ( $p p b)$. Let $\mathrm{p}_{\mathrm{X}}(\mathrm{x})$ denote the probability density function of the random variable $X$, $\mathrm{E}[\mathrm{x}]$ denote the expected value of the containment concentration measured in pbb (i.e., the risk of the ground water being contaminated by an average concentration of TCE), and let the probability density function be discretized into $\mathrm{n}$ regions over the entire universe of contaminant concentrations as is presented by (4):

$$
\left\{f_{1}(x), f_{2}(x), \ldots, f_{n}(x)\right\}
$$

Let $\mathrm{p}_{\mathrm{i}}, \mathrm{i}=1,2, \ldots, \mathrm{n}$, represent the corresponding probabilities of the contamination given by (4), where

$$
\mathrm{p}_{1}+\mathrm{p}_{2}+\ldots+\mathrm{p}_{\mathrm{i}}=1, \mathrm{p}_{\mathrm{i}} \quad 0, \quad \mathrm{i}=1,2, \ldots, \mathrm{n}
$$

Then the expected value of the risk ofTCE contamination of the ground water system is:

$$
E[x]=p_{1} f_{1}(x)+p_{2} f_{2}(x)+p_{3} f_{3}(x)
$$

Integration (instead of summation) can be used in (6) for the continuous case. Clearly, the system of equations (1) to (3) are similar to the system of equations (4) to (6), and in many respects, the expected value of risk is similar in its theoretical-mathematical construct to the commensuration of all costs, benefits, and risks into monetary units as discussed in Section B. In particular, the expected-value operation commensurates contamination (events) of low concentration and high probab ility with contamination of high concentration and low probability. For example, even ts $\mathrm{f}_{1}(\mathrm{x})=2 \mathrm{ppb}$ and $\mathrm{f}_{2}$ $(\mathrm{x})=20,000 \mathrm{ppb}$ with the probabilities $\mathrm{p}_{1}=0.1$ and $\mathrm{p}_{2}=$ 
0.00001 , respectively, yield the same contribution to the overall expected value of risk of contamination:

$$
\mathrm{E}[\mathrm{x}]=(0.1)(2)+(0.00001)(20,000)=0.2+0.2
$$

However, to the decisionmakers in charge, the relatively low likelihood of a disastrous contamination of the ground water system with 20,000 ppb of TCE cannot be equivalent to contamination at a low concentration of 0.2 ppb, even with a very high likelihood of such contamination. Due to the nature of mathematical smoothing, the averaging function of the contaminant concentration in this example does not lend itself to prudent management decisions. This is because the expected value of risk does not accentuate the catastroph ic events and their consequences, thus misrepresenting what would be perceived as unacceptable risk.

It is worth noting that despite the number of "good" decisions managers make during their tenure, they are likely to be penalized for any disastrous decisions they make, no matter how few. The notion of "not on my watch" stems from this truism. In this and other senses, the expected value of risk fails to represent a measure that truly com mun icates the m anag er's or the decisionmaker's intentions and perceptions. The conditional expected value of the risk of extreme events generated by the partitioned multiobjective risk method (PMRM), when used in conjunction with the (unconditional) expected value, can markedly contribute to the total riskmanagement approach (Asbeck and Haimes 1984, Haimes 1998). A conditional expectation is defined as the expected value of a random variable given that this value lies within some prespecified range. In this case, the decisionmakers must make trade-offs not only between the cost of preventing TCE contamination vs. the expected value of such risk, but also between the cost of preventing contamination vs. the conditional expected value of risk of an extreme level of TCE contamination. Such a dual multiobjective analysis provides the manager with more complete, more factual, and less-aggregated information about all viable policy options and their associated tradeoffs. The conditional expected value of risk has been widely applied to dam safety and to numerous other studies.

\section{PRESENT VALUE OF MONEY}

Most, if not all, economic analyses of water resources systems make use of the present value of money to bring to a common denom inator funds expended or received at different time periods. Here again, while the present- value concep $t$ can be a valuable $m$ etric, it has been applied indiscrim inately across the board. In its core, the presentvalue metric commensurates dollars of different values into one index through the discount rate. For a lender, the discount rate used in such analyses is intended to account for the opportunity loss of the use ofmoney lent to others, the risk of loss of the funds, and the rate of inflation, among others. The multidimensional characteristics of the discount rate coupled with the use of a fixed value in the commensuration process, raise a serious question about the proper and unconditional use of the present-value as a universal metric.

Let $f_{i}(x)$ represe $n t$ a stream of $n$ expenditures over $n$ time periods, $i=1,2, \ldots, n$, where $x$ represents a set of policy options. Let $r$ represent the discount rate used in the analysis. Then, the present value of cost (PVC) for the entire stream of expenditures is given by Equation (8):

$$
\begin{aligned}
& \text { PVC }=(1+r)^{-1} f_{1}(x)+(1+r)^{-2} f_{2}(x)+\ldots \\
& +(1+r)^{-n} f_{n}(x) \\
& \text { Let } p_{i}=(1+r)^{-i} / \quad(1+r)^{-i}, i=1,2, \ldots, n
\end{aligned}
$$

Where $\mathrm{p}_{\mathrm{i}}=1$, and $\mathrm{p}_{\mathrm{i}} \quad 0$

Then, Equation (8) can be rewritten as

$$
P V C=p_{1} f_{1}(x)+p_{2} f_{2}(x)+p_{3} f_{3}(x)
$$

Since the system of Equations (2) and (11) are similar in their mathem atical construct, argum ents made in Section $\mathrm{B}$ againstcommensurating the objectives by the averaging process, have some validity here as well. In essence, the present value of monetary costs and benefits constitutes a single metric that attempts to respond to the inherent complexity and multiple purposes of water and related land systems discussed in Section A. Clearly, the un its and dimensions of the expected value of risk are different from those of the present value of cost or benefit; nevertheless, the commensuration process is the same. The limitation of the commensuration process in the present value metric is often magnified when sustainable development is of prime concern. In evaluating the effects of investments on the regional environment, ecology, and socio economic well being, using the same discount rate without discrimination is an implicit act of precommensurating objectives of different units through the convenience of the weighting approach. Finally, many argue that variable discount rates should be used for different periods; however, this practice is not com monly followed bec ause of the new problems that it introduces. 


\section{RELIABILITY ANALYSIS AS A SURROGATE FOR RISK A NAL YSIS}

The literature offers some confusion about the terms risk and uncerta inty, and this necessitates a restatement here of their conventional definitions: the term risk commonly refers to a situation in which the potential outcomes can be described in objectively known probability distributions. The term uncertainty commonly refers to a situation in which no reasonable probabilities can be assigned to the potential outcomes. Uncertainty is the inability to determine the true state of affairs of a system; it can be caused by incomplete knowledge or stochastic variability. Uncertainty caused by variability is a result of inherent fluctuations or differences in the quantity of concern. More precisely, variability occurs when the quantity of concern is not a specific value but rather a population of values (Haimes 1998).

While reliability modeling has proven its usefulness for designing and maintaining water infrastructures, the following truths are often ig nored in practice: (i) complex systems more often have not only one, but any number of paths to failure, and (ii) to know the consequences of failures is at least as important as to know failure likelihoods. Thus, the distinction between reliability and risk is not merely a semantic issue; rather, it is a major element in resource allocation and management decisions throughout the life cycle of water resources systems (whether in design, construction, operation, maintenance, or replacement). Risk was defined earlier as a measure of the probability and severity of adverse effects. Unreliability, is only a measure of the probability that the system does not meet its intended functions. In other words, unreliability does not include the consequences of failures, whereas risk as measure of the probability and consequences of the adverse effects, is inclusive and thus more representative. Clearly, not all failures can justifiably be prevented at all costs. Thus, system reliability cannot constitute a viable metric for resource allocation, unless an a priori level of reliability has been determined. This bring s us to the du ality between risk and reliability on the one hand, and multiple objectives and a single objective optimization on the other.

In the multiple-objective model, the level of acceptable reliability is associated with the corresponding consequences (i.e., constituting a risk mea sure) and is thus traded off with the associated cost that would reduce the risk (i.e., improve the reliab ility and/or reduce the adverse effects). In the single-objective model, on the other hand, the level of acceptable reliability is not explicitly associated with the co rrespon ding conseque nces; rathe $r$ it is often predetermined by individuals who become anonymous over the years (or the reliability is parametrically evaluated) and thus is considered as a constraint in the model.

There are, of course, both historical and evolutionary reasons as well as substantive and functional justifications for the more common use of reliability analysis rather than risk analysis. Historically, engineers have always been concerned with strength of materials, durability of product, safety, surety, and ope rability of various system s. The concept of risk as a quantitative measure of both the probability and consequences (or adverse effects) of a failure has evolved relatively recently. From the substantive-functional perspective, however, many engineers or decisionmakers cannot relate to amalgamating two diverse concepts with differen t units probabilities and consequences - into one concept termed risk. Nor do they accept the metric with which risk is commonly measured. The common metric for risk (as discussed earlier - the expected value of an adverse outcome) essentially commensurates events of low probability and high consequences with those of high probab ility and low consequences. In this sense, one may find basic philosophical justifications for en gineers to avoid using the risk metric and instead work with reliability. Furthermore and most importantly, dealing with reliability does not require the engineer to make explicit trade-offs between cost and the outcome resulting from structural or product failure. Thus, design engineers isolate themselves from the social consequences that are byproducts of the trade-o ffs betwe en reliability and cost. The design of levees for flood protection may clarify this point furth er.

Designating a "one-hundred-year return period" means that the engineer will design a flood-protection levee for a predetermined water level that on the average is not expected to be exceeded more than once every hundred years. Here, ignoring the soc ioeconom ic consequen ces, e.g., loss of lives and property damage due to a high water-level that might exceed the one-hundred-year return period, the design engineers shield themselves from the broader issues, such as risk to the population's well-being. On the other hand, addressing the multiobjective dimension that the risk metric brings requires much closer interaction and coordination between design engineers and decisionmak ers. In this case, an interactive process is required to reach acceptable levels of risks, costs, and benefits. In a nutshell, complex water resources issues, especially those involving public policy with health and socioeconomic dimensions, should not be addressed through overly simplified models and metrics. With the increasing reliance on supervisory control and data acquisition (SCADA) systems in water resources 
management, and as the demarcation line between hardware and software slowly, butsurely, fadesaway, and with the ever-evolving and increasing role of design engineers and systems analysts in technology-based decisionmaking, a new paradigm shift is emerging. Th is shift is characterized by a strong overlapping of the responsibilities of engineers, executives, and lesstechnically-trained water systems ma nagers.

The likelihood of multiple or comp ound fa ilure modes in water resources systems (as well as in other physical systems) adds another dimension to the limitations of a single reliability metric for a water infrastructure (Park et al., 1998, Lambert et al., 1996). Indeed, because one must address multiple reliabilities of a system, the need for explicit trade-offs among risks and costs becomes more critical. Compound failure modes are defined as two or more paths to failure with consequences that depend on the occurrence of combinations of failure paths. Consider the following examples: (a) a water distribution system, which can fail to provide adequate pressure, flow volume, water quality, and other needs; (b) the navigation channel of an inland waterway, which can fail by exceeding the dredge capacity and by closing to barge traffic; and (c) highway bridges, where failure can occur from deterioration of the bridge deck, corrosion or fatigue of structural elements, or an external loading such as flood. Water quality could be used as another basis for the reliability of the water distribution system. None of these failure modes is independent of the others in probability or consequence. For example, deck cracking can contribute to structural corrosion. Structural deterioration in turn can increase the vulnerability of the bridge to floods; nevertheless, the individual failure modes of bridges are typically analyzed in isolation of one another. Acknowledging the need for multiple metrics of reliability of capacity, pressure, hydraulic capacity (joint requirem ents for flow volume and pressure in the system), or quality could markedly improve decisions regarding maintenance and rehabilitation, especially when these multiple reliabilities are aug mented with risk metrics.

\section{THE FALLACY OF OPTIMIZATION}

Since metrics and systems modeling are the focus of th is paper, it seems appropriate to briefly address the "solution" of models: namely, systems optimization. Quantitative analysis in water-resource systems engineering heavily re lies on mathematical models, which in turn, are assumed to represent reasonably well the essence of the water system under study. The objective function (or functions in multiobjective analysis) is often the driving force in these models, and any "optimal" solution derived is clearly dependent on the assumptions that are embedded in the representation of the objective functions, constraints, and inpu t-output relationships. The term "optimal solution" essentially refers to the best solution of the mathem atical model un der all assumptions, whether explicitly assumed, intentionally excluded, or inadvertently omitted. Clearly, the model optimal solution may be far from, or totally unrelated to the actual system's optimal solution. Then how should mathematical models be used as a valuable tool in the decisionmaking process?

Obviously, mathematicalmodels should notsubstitute for the decisionmaking process; rather, they are a tool. They can be very valuable in generating future possible outcomes under certain conditions and assump tions. In multiobjective analysis, where the concept of optimality is expanded into Pareto optimality, generating model Pareto optimal plans can be invaluable in identifying specific characteristics and attributes of the water system. In sum, recognizing that the term "optimal solution" pertains on ly to the model and not necessarily to the real system would help diffuse some of the misgivings among practitioners and help to develop a more sober attitude on the part of the modelers and a nalysts.

\section{EPILOGUE}

During the past three decades the consideration of multiple objectives in modeling and decisionmaking has grown by leaps and bounds. The eighties in particular have seen the emphasis shift from the dominance of single-objective modeling and optimization toward an emphasis on multiple objectives. In particular, multiobjective analysis has emerged as a philosophy that integrates common sense with empirical, quantitative, normative, descriptive, and value-judgment-based analysis. It is a philosophy that is supported by advanced systems concepts (e.g., data management procedures, modeling methodologies, optimization and simulation techniques, and decisionmaking approaches) that are grounded on both the arts and the sciences for the ultim ate purpose of improving the decision making process.

Modeling constitutes the road map that guides the analyst throughout the journey of water-resources planning, design, and management, and it may be viewed through many spectacles depending on the analyst's perspectives, vision, and circumstances. Metrics are the building blocks of modeling; therefore, their appropriateness and representativeness in any specific model are the sine qua non for good modeling and ultimately as an effective tool for decisionmaking. In particular, the optimum doesn't exist in an objective sense per se. An "optimum" solution to a real-life problem depends on myriad factors, which include who the decisionmakers are, what their perspectives are, what the biases of the modeler are, what 
the credibility of the database is, etc. Therefore, a mathematical optimum to a model does not necessarily correspond to the optimum for the real-life problem.

This article is an attempt to highlight some of the flaws in the metrics used in modeling, focusing on recognizing the importance of multiobjective modeling and ana lysis. This argument is particularly critical in risk assessment and management, where trade-offs among all important and relevant costs, benefits, and risks must be considered within a multiobjective fram ework. Today, with manmade hazards such as terrorism and cyber-tampering added to natural threats such as floods and earthquakes, analyzing risk within a multiobjective framework is not just an option - it is imperative.

\section{REFERENCES}

Asbeck, Eric, and Yacov Y. Haimes. 1984. "The Partitioned Multiobjective Risk Method," Large Scale Systems, Vol.6, No.1, 13-38.
Haimes, Yacov Y. 1977. Hierarchical Analyses of Water Resources Systems: Modeling and Optimization of Large-Scale Systems. McGraw-Hill Book Company, NY.

Haimes, Yacov Y. 1998. Risk Modeling, Assessment, and Management, John Wiley \& Sons, NY.

Lambert, James H., Lori R. Johnson, and Yacov Y. Haimes. 1996. "The Impact of Multiple Failure Modes in Risk Analysis for Civil Infrastructure Mana gement," Risk-Based Decision Making in Water Resources VII, Y. Y.

Haimes, D. A. Moser, and E. Z. Stakhiv. Ed. American Society of Civil En gineers, NY.

Park, Joshua I., James H. Lamb ert, and Yaco v Y. Haim es. 1998. "Hydraulic Power Capacity of Water Distribution Networks in Uncertain Conditions of Deterioration," Water Resources Research, Vol. 34, No. 12, pp.3605-3614.

This article is dedicated to the memory of Warren A. Hall, one of the principal founders of UCOWR. 